\title{
Features of Hypertension and Coronary Heart Disease Secondary Prevention in the Population of Veliky Novgorod Russia
}

\author{
Zhbanova N*, Yuhno M, and Prosorova I \\ Department of Education and Science, Russia
}

*Corresponding author : Zhbanova N, Department of Education and Science, The-Wise Novgorod State University, Russia.

Received Date: December 17, 2018

Published Date: January 04, 2019

Keywords: Secondary prevention of hypertension/CAD; Regional characteristics

\section{Objective}

To assess regional features of secondary prevention, as well as compliance with modern standards of treatment of patients with hypertension and coronary artery disease.

\section{Material and Methods}

By analogy with the "Regular Treatment and Prevention as The Key to Improving CVD Situation in Russia - RELIEF" large-scale study on assessing the adequacy of the treatment of hypertension and coronary heart disease and adherence of patients to the doctor's recommendations, in a period from 2012 to 2014 onetime, continuous, clinical and epidemiological study was conducted among 39 first contact physicians in Veliky Novgorod hospitals and 212 patients with a verified diagnosis of hypertension and coronary heart disease. Thirtyseven women and 73 men with the diagnosis of coronary artery disease were selected and 33 women and 69 men diagnosed with myocardial infarction. All patients were examined clinically taking into account biochemical and functional parameters. To assess the quality of treatment of patients, an open, non-experimental, descriptive, cross-sectional clinical and pharmaceutical research of doctors' prescriptions and receiving medications by patients, was conducted. Each patient had two medical record cards. One card was filled in by a doctor - "Doctor's Card: Patient's Conditions"; the second - by a patient - "Patient's card". The structure of such cards was tested in the course of a federal research in 2006. Statistical and mathematical calculations were performed using Statistica 10 (Russian- language version, 2012) licensed programme.

\section{Result}

During this study it was found that ambulatory physicians most often prescribed ACE inhibitors to treat hypertension (28.6\% of cases). As for other classes of drugs, the doctors preferred $ß$ - blockers (20.7\% of cases), calcium antagonists - ACC (15.5\%) and sartans (13.8\%). Considering $\beta$ - blockers as one of the leading groups in cardiology today, the absolute priority was given to prolonged, cardioselective medications with no intrinsic sympathomimetic activity. Diuretics as monotherapy (hydrochlorothiazide, indapamide) were administered in $19.7 \%$ of cases, they were usually used in the form of rational combination with other medications in order to obtain additional hypotensive effect and reduce side effects. Antihypertensive drugs of other ARA II groups, imidazoline receptor agonists, alpha - blockers (1.7\% of cases) were much less frequently prescribed. Isolated cases of monotherapy administering of such hypertension drugs as Rauwolfia, reserpine, clonidine, Papazol (0.5\%) were noted.

Combination therapy according to $51 \%$ of doctors, should be used as first-line therapy for hypertension, because it has several advantages over monotherapy, in particular, combination therapy provides a better antihypertensive effect in low doses and therefore has a lower incidence of side effects and better patient adherence. The other group of doctors (49\%) thought that during the transition to combination, compliance sharply decreases. However, in order to achieve target blood pressure in patients of outpatient care, only $12 \%$ of patients needed the appointment of a single drug, $54 \%$ needed two, 34\% - 3 and more drugs. 


\section{Conclusion}

In assessing the effectiveness of secondary prevention of hypertension / CHD in Veliky Novgorod it was found that prescriptions profile has statistically significant differences ( $p$ $=0.0012$ ), depending on the qualifications of the doctor and the patient's adherence to treatment. There is no unified system in the frequency of receiving medicaions of virtually all groups during monotherapy. At the same time, patients comply with specialists recommendations of receiving ACE inhibitor drugs, beta-blockers, Sartana and recommendations on receiving ACC drugs and diuretics from local doctors or family physicians. The general principles are identified only in combination therapy with a combination of ACE inhibitors and ACC, the frequency of its administering does not exceed $1.5-2 \%$. Frequency of its administering in coronary heart disease (in groups): beta-blockers-20.5\%; desagregants -18.1\%; nitrates - 17,7\%; statins -13.9\%; ACE inhibitors -12.6\%; diuretics $-4.2 \%$; ACC - 3.8\%; cardioprotectors - 3.4\%; Sartana - 1.6\%. The frequency of administration of drugs from other groups does not exceed $0.8 \%$. Frequency of administering during hypertension (in groups): ACEI - 28.6\%; beta-blockers-20.7\%; diuretics - 19.7; Sartana -13.8\%; ACC - 15.5\% and AIR - 1.7\%. A combination of ACE inhibitors in ACC was applied in $35.7 \%$ of cases during combined therapy; ACE inhibitors + diuretics in $28.6 \%$ of cases and Sartana +diuretics in $35.7 \%$ of cases. The structure of the products was significantly different from that of medications in the Northwestern Federal District. Prescription frequency and receiving the drugs frequency was somewhat higher than in Northwestern Federal District only in group of sartans.

\section{Acknowledgement}

None.

\section{Conflict of Interest}

No conflict of interest. 